\title{
Thymoquinone induces apoptosis in human colon cancer HCT116 cells through inactivation of STAT3 by blocking JAK2- and Src-mediated phosphorylation of EGF receptor tyrosine kinase
}

\author{
JUTHIKA KUNDU ${ }^{1}$, BU YOUNG CHOI ${ }^{2}, \mathrm{CHUL}-\mathrm{HO} \mathrm{JEONG}^{1}$, JOYDEB KUMAR KUNDU ${ }^{1}$ and KYUNG-SOO CHUN ${ }^{1}$ \\ ${ }^{1}$ College of Pharmacy, Keimyung University, Dalseo-Gu, Daegu 704-701; ${ }^{2}$ Department of Pharmaceutical Science \\ and Engineering, Seowon University, Cheongju, Chungbuk 361-7472, Republic of Korea
}

Received February 13, 2014; Accepted April 22, 2014

DOI: $10.3892 /$ or.2014.3223

\begin{abstract}
Thymoquinone (TQ), a compound isolated from black seed oil (Nigella sativa), has been reported to possess anti-inflammatory and anticancer activities. However, the molecular mechanisms underlying the anticancer effects of TQ remain poorly understood. In the present study, we found that TQ significantly reduced the viability of human colon cancer HCT116 cells in a concentration- and time-dependent manner. Treatment of cells with TQ induced apoptosis, which was associated with the upregulation of Bax and inhibition of Bcl-2 and Bcl-xl expression. TQ also activated caspase-9,-7, and -3 , and induced the cleavage of poly-(ADP-ribose) polymerase (PARP). Pretreatment with a pan-caspase inhibitor, z-VAD-fmk, abrogated TQ-induced apoptosis by blocking the cleavage of caspase- 3 and PARP. Treatment of cells with TQ also diminished the constitutive phosphorylation, nuclear localization and the reporter gene activity of signal transducer and activator of transcription-3 (STAT3). TQ attenuated the expression of STAT3 target gene products, such as survivin, c-Myc, and cyclin-D1, -D2, and enhanced the expression of cell cycle inhibitory proteins p27 and p21. Treatment with TQ attenuated the phosphorylation of upstream kinases, such as Janus-activated kinase-2 (JAK2), Src kinase and epidermal growth factor receptor (EGFR) tyrosine kinase. Pharmacological inhibition of JAK2 and Src blunted tyrosine phosphorylation of EGFR and STAT3, while treatment with an EGFR tyrosine kinase inhibitor gefitinib inhibited phosphorylation of STAT3 without affecting that of JAK2 and Src in HCT116 cells. Collectively, our study revealed that TQ induced apoptosis in HCT116 cells by blocking STAT3 signaling via
\end{abstract}

Correspondence to: Professor Kyung-Soo Chun, College of Pharmacy, Keimyung University, 1095 Dalgubeoldaero, Dalseo-Gu, Daegu 704-701, Republic of Korea

E-mail: chunks@kmu.ac.kr

Key words: thymoquinone, HCT116 cells, apoptosis, STAT3, EGFR inhibition of JAK2- and Src-mediated phosphorylation of EGFR tyrosine kinase.

\section{Introduction}

Colorectal cancer is one of the leading causes of mortality and ranks among the three most common cancers in developed countries $(1,2)$. More than one million new cases of colorectal cancer are diagnosed every year (3). Multiple lines of evidence suggest that bioactive compounds present in various edible and medicinal plants can prevent colon carcinogenesis (4). Thymoquinone (TQ; Fig. 1A), a dietary phytochemical, is the major bioactive constituent present in black seed oil (Nigella sativa), which is widely consumed as a condiment and has long been used in Ayurvedic medicine. TQ has been reported to exert antioxidative, anti-inflammatory and anticancer effects (5-7). Several studies have reported that TQ inhibits cell proliferation, induces apoptosis and impedes the in vivo growth of xenograft tumors of various human cancer cells including those of the stomach $(8,9)$, colorectal $(10,11)$, lungs (12), breast (5) and prostate (13). In a recent study, TQ was shown to inhibit 1,2-dimethylhydrazine-induced initiation and promotion of colon carcinogenesis in Wister rats (14). However, the molecular basis of the anticancer effects of TQ in colon cancer has yet to be fully elucidated.

Evasion from apoptosis is one of the hallmarks of cancer (15). Tumor cells bypass the apoptotic process following two biochemical pathways (16). The intrinsic or mitochondriamediated pathway of apoptosis involves the depolarization of mitochondrial membrane, release of cytochrome $c$, sequential activation of caspase-9, -7 and -3, and the cleavage of poly(ADP-ribose) polymerase (PARP). The extrinsic pathway, on the other hand, is mediated through the activation of cell membrane-bound death receptors, followed by the activation of pro-caspase- 8 , which then execute cell death by triggering the activity of caspase-3 $(16,17)$. According to the intrinsic mechanism of apoptosis induction, the mitochondrial membrane potential is largely regulated by relative abundance of different B-cell lymphoma (Bcl) family proteins. Whereas localization of Bcl-2 and Bcl-xl proteins stabilizes mitochondrial membrane integrity, downregulation of Bcl-2 and concomitant over- 
expression and subsequent translocation of proapoptotic protein Bax to the mitochondria lead to mitochondrial membrane depolarization and release of cytochrome $c$, which triggers the activation caspase cascade, thereby inducing cell death $(16,17)$.

Bcl-2 and Bcl-xl are oncoproteins that are frequently overexpressed in many cancers (16). One of the transcriptional regulators of $\mathrm{Bcl}-2$ family proteins is signal transducer and activator of transcription-3 (STAT3), which is a latent transcription factor that normally resides in the cytoplasm (18). In response to diverse growth stimulatory signals, STAT3 gets activated through phosphorylation by upstream kinases, such as Janus-activated kinase-2 (JAK2) (19) and Src tyrosine kinase (20) followed by STAT3 dimerization and nuclear localization. The oncogenic signal transduction pathway mediated through phosphorylation of epidermal growth factor receptor (EGFR) tyrosine kinases also transmits activating signals to STAT3 $(21,22)$. While transient activation of STAT3 is associated with the growth and development of various organs, constitutive activation of STAT3 has been implicated in carcinogenesis (23). The activation of STAT3 not only assists tumor cells to evade apoptosis but also promotes cell proliferation by transactivating the genes encoding various cell survival proteins, such as cyclins, c-Myc and survivin $(24,25)$. The blockade of inappropriate activation of STAT3 signaling inhibits cell proliferation and induces apoptosis. Thus, STAT3 appears as a molecular target of many anticancer agents. Herein, we report that TQ induces apoptosis in HCT116 cells through the inhibition of STAT3 signaling pathway by blocking the phosphorylation of EGFR tyrosine kinase via modulation of JAK2 and Src kinases.

\section{Materials and methods}

Materials. TQ (purity 99\%), z-VAD-fmk and $\beta$-actin antibody were purchased from Sigma-Aldrich (St. Louis, MO, USA). AG490 and PP2 were purchased from Cayman Chemical Co. (Ann Arbor, MI, USA). Gefitinib was purchased from LC Laboratories (Woburn, MA, USA) and antibodies against cleaved caspase-9, -3, and -7, PARP, Bcl-2, Bcl-xl, Bax, STAT3, p-STAT3 (Y705), JAK2, p-JAK2, Src, p-Src, EGFR, p-EGFR (Y1173), cyclin-D1, -D2, and survivin were obtained from Cell Signaling Technology Inc. (Beverly, MA, USA). Primary antibodies against each of c-Myc, p27, p21, lamin A and horseradish peroxidase-conjugated secondary antibodies were purchased from Santa Cruz Biotechnology (Santa Cruz, CA, USA). All other chemicals were of analytical or highest purity grade available.

Cell culture and treatment. HCT116 cells were obtained from American Type Culture Collection (ATCC) and maintained in RPMI-1640 medium supplemented with $10 \%$ fetal bovine serum and antibiotics $(100 \mathrm{U} / \mathrm{ml}$ penicillin and $100 \mu \mathrm{g} / \mathrm{ml}$ streptomycin) at $37^{\circ} \mathrm{C}$ in a humidified incubator containing $5 \% \mathrm{CO}_{2}$ and $95 \%$ air. In all the experiments, cells were seeded at $2 \times 10^{5}$ cells $/ \mathrm{ml}$ and treated with TQ at 50-60\% confluence. All chemicals were dissolved in ethanol.

Cell proliferation assay. The anti-proliferative effect of TQ against HCT116 cells was measured by using a solution of tetrazolium compound [3-(4,5-dimethylthiazol-2-yl)-5(3-carboxymethoxyphenyl)-2-(4-sulfophenyl)-2H-tetrazolium, inner salt (MTS) (Promega, Madison, WI, USA). Briefly, cells $\left(2 \times 10^{3}\right)$ were incubated in triplicate in a 96-well plate in the presence or absence of TQ in a final volume of $0.1 \mathrm{ml}$ for different time intervals at $37^{\circ} \mathrm{C}$. Thereafter, $20 \mu \mathrm{l}$ of MTS solution was added to each well and incubated for $60 \mathrm{~min}$. The number of viable cells was measured in a 96-well plate at an optical density of $492 \mathrm{~nm}$ on a microplate reader (Tecan Trading AG, Männedorf, Switzerland). Cell viability was described as the relative percentage of control.

Annexin $V$ staining. Annexin $\mathrm{V}$ staining was performed using FITC-Annexin V staining kit (BD Biosciences, San Jose, CA, USA) following the manufacturer's instructions. Briefly, TQ-treated cells were washed with PBS and resuspended in binding buffer containing Annexin $\mathrm{V}$ and propidium iodide (PI). Fluorescence intensity was measured using flow cytometry (BD Biosciences).

Western blot analysis. Cells were harvested and lysed with RIPA buffer to obtain whole cell lysate. Collected protein samples were quantified by using bicinchoninic acid protein assay kit (Pierce Biotechnology, Rockford, IL, USA). The cytosolic and nuclear extracts were prepared by using NE-PER Nuclear and Cytoplasmic Extraction Reagent kit (Thermo Scientific, Rockford, IL, USA) according to the procedure described previously (6). Nuclear proteins were collected and stored at $-70^{\circ} \mathrm{C}$ after determination of protein concentration by using Bradford Reagent (Bio-Rad Laboratories, Hercules, CA, USA). The protein samples were separated by sodium dodecyl sulfate-polyacrylamide gel electrophoresis and immunoblot analysis was performed according to the protocol described previously (6). Immunoblot membranes were incubated with SuperSignal Pico Chemiluminescent substrate or Dura Luminol substrate (Thermo Scientific), according to the manufacturer's instructions, and visualized with ImageQuant ${ }^{\mathrm{TM}}$ LAS 4000 (Fujifilm Life Science, Tokyo, Japan).

Caspase-3 activity assay. The activity of caspase-3 was detected using the Caspase-3 Colorimetric Activity Assay kit (Millipore, Billerica, MA, USA). The assay was performed in 96-well plates by incubating cell lysates $(50 \mu \mathrm{g})$ in $100 \mu \mathrm{l}$ reaction buffer containing caspase-3 substrate Ac-DEVD-pNA at $37^{\circ} \mathrm{C}$ for $2 \mathrm{~h}$ and $30 \mathrm{~min}$ according to the manufacturer's protocol.

STAT3-Luciferase reporter gene assay. Cells were seeded into 12-well plates at a density of $5 \times 10^{4}$ cells/well prior to transfection. Cells were transfected with p-STAT3-TA-luc (Clontech, Palo Alto, CA, USA) or control vector using Genefectin transfection reagent (Genetrone Biotech, Seoul, Korea). After $24 \mathrm{~h}$ of transfection, cells were treated with TQ for an additional $24 \mathrm{~h}$ and cell lysis was carried out with $1 \mathrm{X}$ reporter lysis buffer. After mixing the cell lysates with luciferase substrate (Promega), the luciferase activity was measured by using luminometer (Tecan Trading AG). The $\beta$-galactosidase assay was carried out according to the supplier's instructions (Promega Enzyme Assay System) for normalizing the luciferase activity and the results were expressed as fold transactivation. 
A<smiles>CC1=CC(=O)C(C(C)C)=CC1=O</smiles>

B

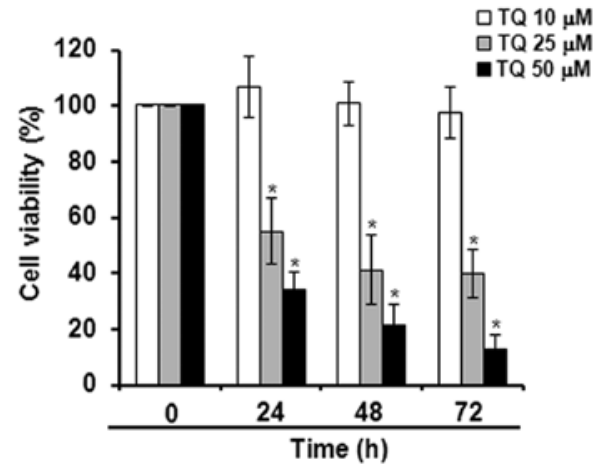

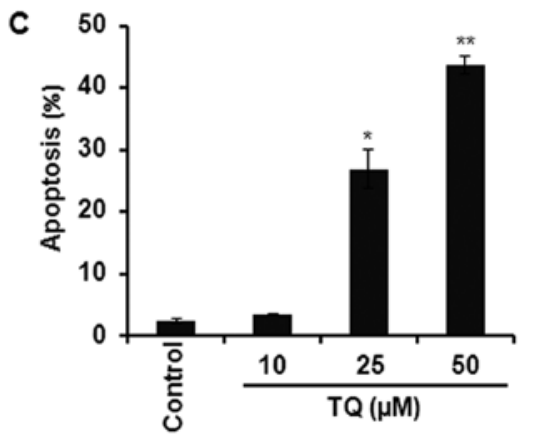

D

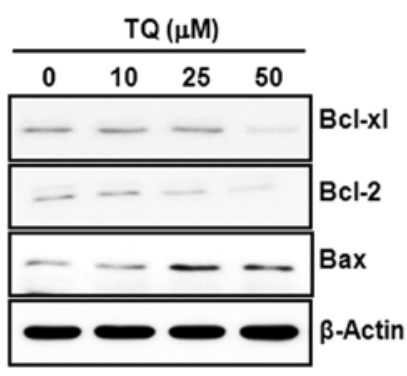

Figure 1. Cytotoxic effect of thymoquinone (TQ) in HCT116 cells. (A) Chemical structure of TQ. (B) HCT116 cells were treated with the indicated concentrations of TQ for 24,48 or $72 \mathrm{~h}$. Cell viability was determined by the MTS assay. Values are expressed as means \pm SD. * $<<0.001$, compared to control. (C) The apoptotic index (\%) was determined by flow cytometry upon treatment of cells with TQ for $24 \mathrm{~h}$ and staining with Annexin V and propidium iodide (PI). Data are representative of three independent experiments. ${ }^{*} \mathrm{p}<0.01$ and ${ }^{* *} \mathrm{p}<0.001$, as compared to control. (D) Western blot analysis of the expression level of Bcl-2, Bcl-xl and Bax upon treatment with TQ for $24 \mathrm{~h}$. Data are representative of three different experiments.

Statistical analysis. When necessary, data are expressed as mean \pm SD of at least three independent experiments, and statistical analysis for single comparison was performed using the Student's t-test. A p-value $<0.05$ was considered to indicate a statistically significant difference.

\section{Results}

TQ induces apoptosis in HCT116 cells. We initially examined the effect of TQ on the viability of HCT116 cells. Incubation of cells with TQ $(10,25$ or $50 \mu \mathrm{M})$ reduced the cell viability in a time- and concentration-dependent manner (Fig. 1B). Annexin V staining of cells treated with the indicated concentrations of TQ showed that the compound induced apoptosis in a concentration-dependent manner (Fig. 1C). Moreover, TQ reduced the expression of anti-apoptotic proteins Bcl-2 and Bcl-xl, while the compound increased expression of proapoptotic protein Bax in HCT116 cells (Fig. 1D).

TQ-induced apoptosis is mediated through caspase activation. Treatment of HCT116 cells with TQ induced the cleavage of caspase-9, -7, and -3, and PARP (Fig. 2A) and increased the caspase-3 activity (Fig. 2B). Pretreatment of cells with a pan-caspase inhibitor z-VAD-fmk abrogated TQ-induced caspase-3 activity (Fig. 2C), and the cleavage of caspase-3 and PARP (Fig. 2D). Moreover, the blockade of caspase-3 activation resulted in the abrogation of TQ-induced apoptosis as assessed by Annexin V staining (Fig. 2E).

$T Q$ attenuates the constitutive activation of STAT3 and expression of its target gene products in HCT116 cells. Since
STAT3 is constitutively active in colon cancer and plays a key role in cell proliferation through transcriptional activation of pro-survival genes (23), we examined the effect of TQ on STAT3 activation in HCT116 cells. Incubation of cells with TQ inhibited constitutive phosphorylation of STAT3 at tyrosine-705 residue (Fig. 3A) and decreased the nuclear localization of p-STAT3 (Fig. 3B). TQ also reduced the STAT3 reporter gene activity in HCT116 cells transiently transfected with STAT3-luc vector (Fig. 3C). Moreover, TQ attenuated the expression of STAT3 target gene products, such as survivin, c-Myc, cyclin-D1, -D2, and increased the expression levels of the cell cycle regulatory proteins p27 and p21 (Fig. 3D).

TQ inhibits STAT3 activation by blocking the phosphorylation of EGFR (Y1173), JAK2 and Src kinases in HCT116 cells. Several upstream kinases, such as EGFR tyrosine kinase $(21,22)$, JAK2 (19), and Src (20), are known to regulate STAT3 activation. We, therefore, examined the effect of TQ on the constitutive activation of these kinases in HCT116 cells. Treatment with TQ markedly diminished the phosphorylation of EGFR at tyrosine-1173 residue (Fig. 4A), JAK2 (Fig. 4B) and Src kinase (Fig. 4C). While JAK2 can directly phosphorylate STAT3, it has been reported that JAK2 (26) and Src (27) function as upstream kinases to EGFR, which transmits activating signals to STAT3 (28). Pretreatment of cells with AG490, a JAK2 inhibitor, attenuated the phosphorylation of EGFR and STAT3 (Fig. 4D). Moreover, pharmacological inhibition of Src kinase by treatment of cells with PP2 diminished the tyrosine phosphorylation of EGFR and STAT3 in HCT116 cells (Fig. 4E). Treatment of cells with gefitinib, an EGFR antagonist, also negated STAT3 phosphorylation 
A

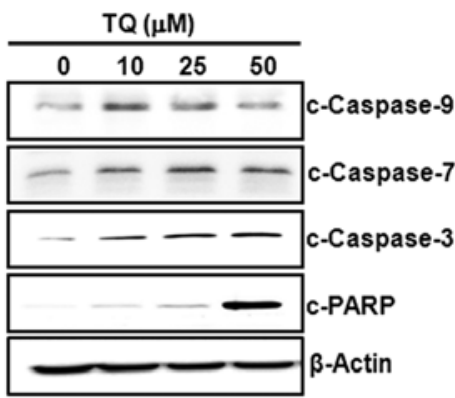

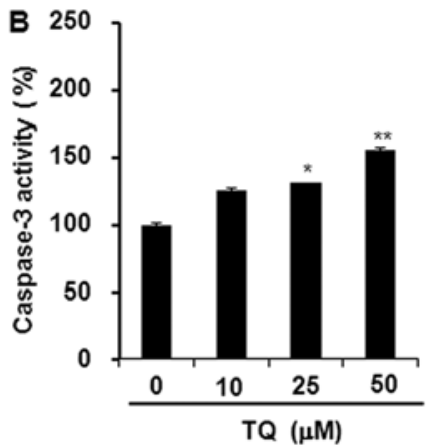
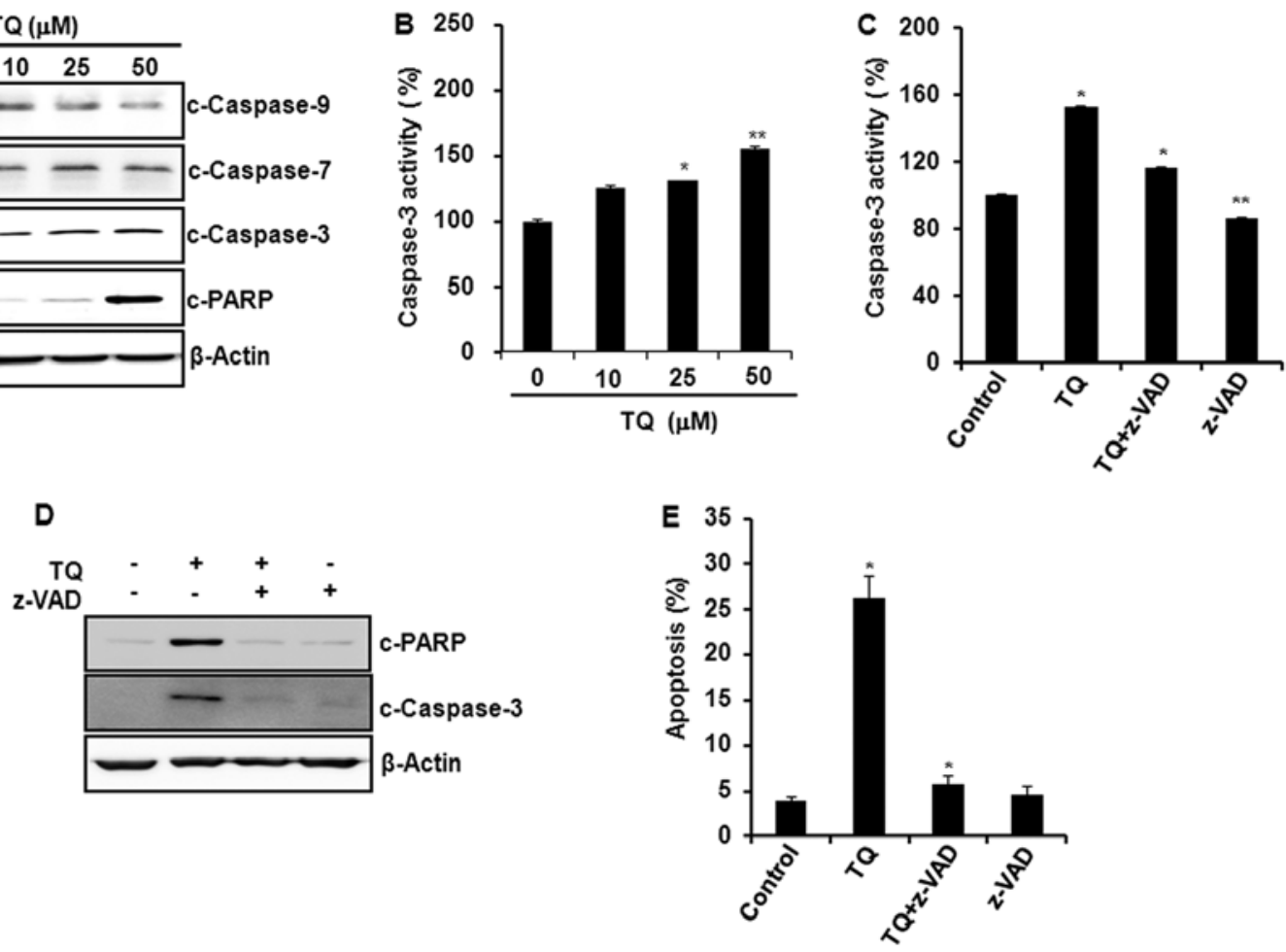

Figure 2. Effect of thymoquinone (TQ) on cellular markers of apoptosis in HCT116 cells. (A and B) Cells were treated with TQ at indicated concentrations for $24 \mathrm{~h}$. (A) Whole cell lysates were subjected to immunoblot analysis for detecting the levels of cleaved caspase-9, -7 and -3 , and cleaved PARP. (B) The caspase-3 activity assay was performed as described in Materials and methods. " $\mathrm{p}<0.05$ and ${ }^{* *} \mathrm{p}<0.01$, as compared to control. (C-E) Cells were treated with or without a pan-caspase inhibitor z-VAD-fmk $(20 \mu \mathrm{M}) 1 \mathrm{~h}$ prior to incubation with TQ $(50 \mu \mathrm{M})$ for $24 \mathrm{~h}$. (C) The caspase-3 activity was measured. ${ }^{*}<<0.01$ (control vs. TQ alone; TQ only vs. TQ plus z-VAD-fmk). (D) Western blot analysis showed the expression levels of cleaved forms of PARP and caspase-3. Data are representative of three different experiments showing a similar trend. $\beta$-actin was used as a loading control. (E) The apoptotic index (\%) was determined by Annexin V staining. " $\mathrm{p}<0.001$ (control vs. TQ alone; TQ only vs. TQ plus z-VAD-fmk).

A

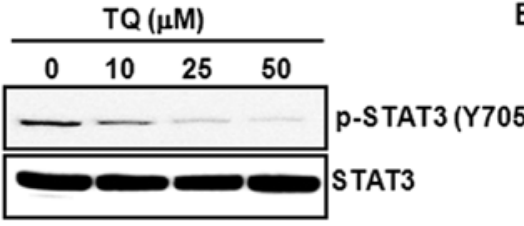

Whole cell lysate

C

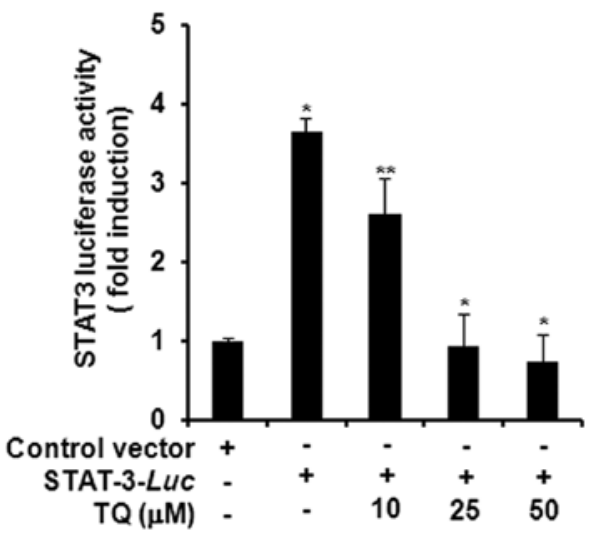

B

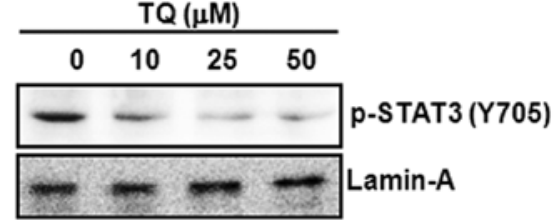

Nuclear extract

D

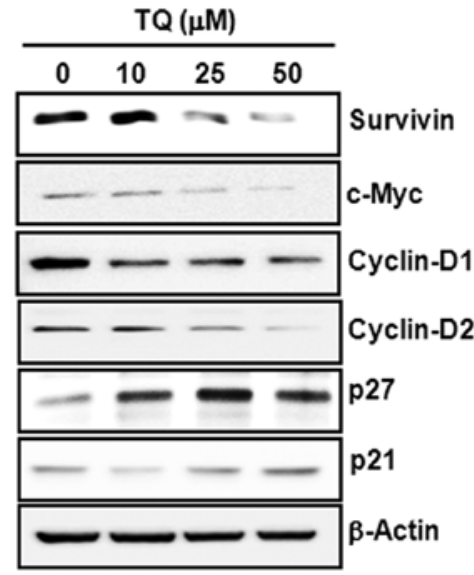

Figure 3. Thymoquinone (TQ) inhibits constitutive activation of STAT3 and expression of its target gene products. (A) Cells were treated with the indicated concentrations of TQ for $24 \mathrm{~h}$ and the level of p-STAT3 (Y705) was detected by immunoblotting. (B) Nuclear extract prepared from cells treated with TQ as indicated above was assessed for nuclear accumulation of p-STAT3 (Y705). (C) Cells were transfected with STAT3-luc construct or control vector and incubated with TQ $(10,25$ or $50 \mu \mathrm{M})$ for $24 \mathrm{~h}$. The luciferase activity was measured as described in Materials and methods. " p $<0.001$ (control vector vs. STAT3-luc vector alone; STAT3-luc vector alone vs. 25 or $50 \mu \mathrm{M}$ TQ plus STAT3-luc vector), ${ }^{* *} \mathrm{p}<0.01$ (STAT3-luc vector alone vs. $10 \mu \mathrm{M}$ TQ plus STAT3-luc vector). (D) Cells were treated with TQ for $24 \mathrm{~h}$ and immunoblot analysis was performed to assess the expression of survivin, c-Myc, cyclin-D1, -D2, p27 and p21. Data represent one of three different experiments showing similar pattern. 
A

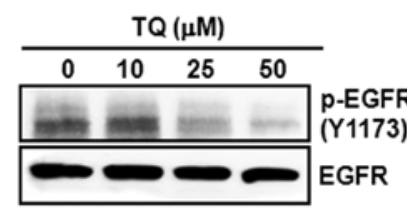

B

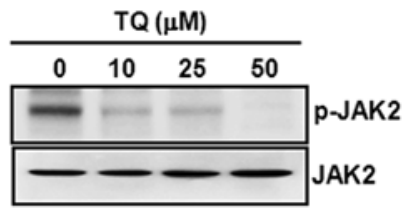

C

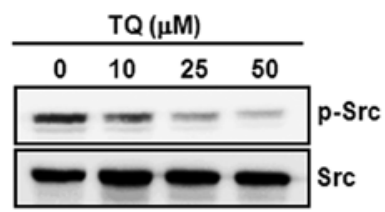

E

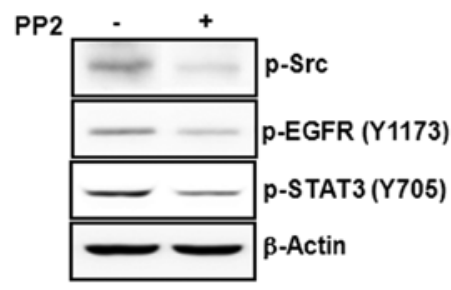

D

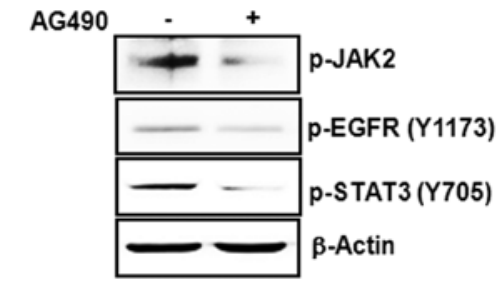

F

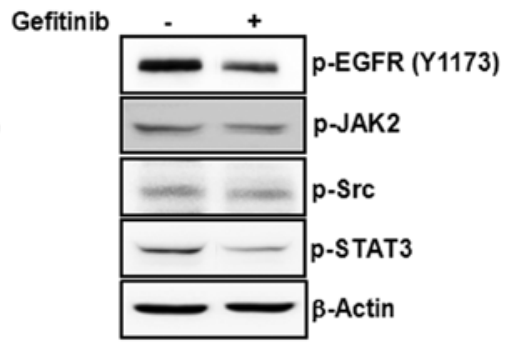

Figure 4. Thymoquinone (TQ) inhibits STAT3 activation by blocking the phosphorylation of EGFR (Y1173), JAK2 and Src kinases. Cells were treated with the indicated concentrations of TQ and the levels of (A) p-EGFR (Y1173), (B) p-JAK2, and (C) p-Src were detected by immunoblotting. Data are representative of three separate experiments exhibiting a similar trend. (D) Cells were treated with or without AG490 (10 $\mu \mathrm{M})$ for $24 \mathrm{~h}$ and the expression of p-JAK2, p-EGFR (Y1173) and p-STAT3 was determined by western blot analysis. (E) Cells were treated with or without PP2 (10 $\mu \mathrm{M})$ for $24 \mathrm{~h}$ and the expression of p-Src, p-EGFR (Y1173) and p-STAT3 was detected. (F) Cells were incubated with or without gefitinib (2 $\mu \mathrm{M})$ for $24 \mathrm{~h}$ and the expression of p-EGFR (Y1173), p-JAK2, p-Src and p-STAT3 was determined by western blot analysis. All data are representative of three independent experiments showing a similar pattern.

without affecting the levels of phosphorylated JAK2 and Src (Fig. 4F).

\section{Discussion}

Despite the marked progress in the development of anticancer therapeutics, cancer remains as a major public health concern worldwide. It has been estimated that the incidence of and mortality from cancer will double in the next 50 years (29). There is now a growing interest in searching for anticancer agents from natural sources, especially those present in edible medicinal plants. Thymoquinone (TQ), the major bioactive constituent of black seed essential oil, has been reported to possess diverse pharmacological activities $(5,30)$. However, the molecular mechanisms of the anticancer activity of TQ have not been fully elucidated. One of the hallmarks of cancer is the ability of tumor cells to evade the apoptosis process and many anticancer therapies have been shown to induce apoptosis selectively in cancer cells.

TQ has been reported to inhibit proliferation and induce apoptosis in human colon cancer cells without exhibiting cytotoxicity to normal human intestinal FHs74Int cells (31). According to this study, TQ-induced cytotoxicity in DLD-1 colon cancer cells was associated with the generation of reactive oxygen species, activation of extracellular signalregulated kinase and c-Jun-N-terminal kinase, and the cleavage of caspase-7 and -3. Moreover, TQ induced apoptosis and G1 phase cell cycle arrest in HCT116 colon cancer cells by increasing the expression of a cell cycle inhibitory protein $\mathrm{p} 21$ with concomitant inhibition of anti-apoptotic protein Bcl-2 (10) and the inactivation of stress response pathway sensor CHEK1 (32) in a p53-dependent manner. In accordance with these previous reports, our study revealed that TQ decreased the cell viability and induced apoptosis in HCT116 cells. Herein, we provide new mechanistic findings that TQ induced apoptosis in HCT116 cells through the downregulation of constitutive activation of STAT3 signaling via inhibition of EGFR tyrosine kinase by blocking phosphorylation of upstream JAK2 and Src kinases (Fig. 5).

The induction of apoptosis by TQ was associated with decreased expression of anti-apoptotic proteins $\mathrm{Bcl}-2$ and $\mathrm{Bcl}-\mathrm{xl}$, and the elevated expression of proapoptotic protein Bax. Moreover, TQ treatment caused the cleavage of caspase-9, -7 and -3 , and induced the caspase- 3 activity followed by the cleavage of PARP. Pretreatment of cells with a pan-caspase inhibitor z-VAD-fmk abrogated TQ-induced apoptosis by blocking the caspase-3 activity and the cleavage of caspase- 3 and PARP. Since caspase- 9 and -7 are initiator caspases in mitochondria-mediated death signaling (16), the activation of caspase-3 and the cleavage of PARP suggest that TQ may induce apoptosis through the activation of the intrinsic pathway. Contrary to our findings, TQ failed to induce the caspase activity and treatment with z-VAD-fmk was unable to block TQ-induced apoptosis in human prostate cancer (PC3) cells (33), suggesting that the mechanisms of apoptosis induction in cancer cells by TQ are variable depending on the cell type. A recent study reported that TQ increased the expression of death receptor-5 (DR5) in neuroblastoma cells, but 


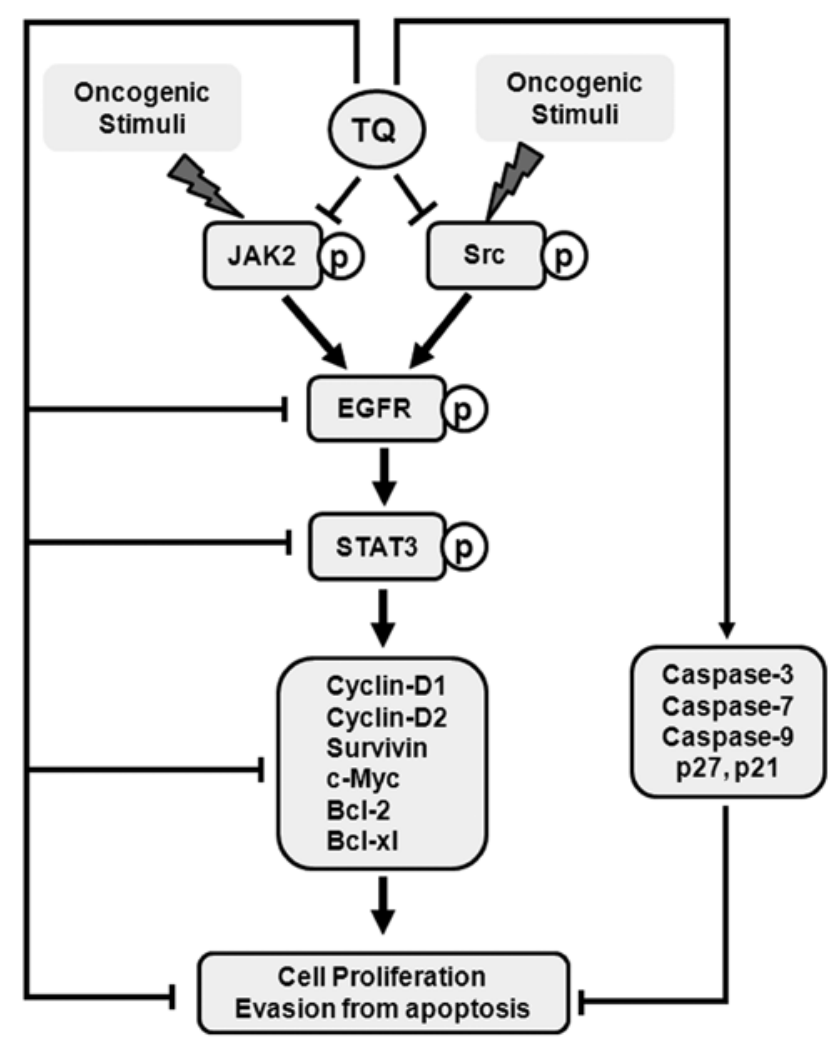

Figure 5. Schematic model showing the molecular mechanisms of thymoquinone (TQ) in inducing apoptosis in HCT116 cells.

the upregulation of DR5 was not associated with TQ-induced apoptosis (34). Whether TQ-induced apoptosis in HCT116 cells is associated with the activation of DR-mediated pathway has yet to be investigated.

It has been reported that $\mathrm{Bcl}-2$ is overexpressed in colon cancer (35) and the Bcl-2-mediated inhibition of apoptosis restores the tumorigenicity of spontaneously regressed colon tumors in vivo (36). The inhibition of Bcl-2 and $\mathrm{Bcl}-\mathrm{xl}$ expression and the increase in Bax protein level, thus, provide a mechanistic basis of apoptosis induction by TQ. The expression of anti-apoptotic proteins Bcl-2 and Bcl-xl is transcriptionally regulated by STAT3 (25), which is overexpressed in colon cancer $(24,37)$. Persistent STAT3 activation increases the proliferation of colon cancer cells in culture and enhances the growth of colon cancer cell xenograft tumors in nude mice (38). Moreover, the inhibition of STAT3 signaling induces apoptosis and cell cycle arrest in colon carcinoma cells (39).

We, therefore, examined the effect of TQ on STAT3 activation in HCT116 cells and, for the first time, we found that TQ suppressed the constitutive activation of STAT3 signaling in human colon cancer HCT116 cells. Our findings that TQ inhibited the constitutive phosphorylation of STAT3 at tyrosine-705 residue and decreased the STAT3 reporter gene activity in HCT116 cells are in good co-relation with previous studies reporting that TQ inhibited proliferation and induced apoptosis in multiple myeloma cells by blocking the activation of STAT3 $(40,41)$. It has been reported that treatment with a STAT3 decoy oligonucleotide diminished the expression of Bcl-2 and activated caspase-3, thereby resulting in the induction of apoptosis in ovarian cancer cell xenograft tumors (42).
Thus, TQ-induced apoptosis in HCT116 cells is mediated, at least in part, by the blockade of STAT3 activation. This was further supported by the findings that TQ inhibited the expression of D-series cyclins, c-Myc and survivin, which are STAT3 target gene products $(25,43)$.

While survivin inhibits apoptosis in cancer cells (44), cyclins (45) and c-Myc (46) promote tumor cell proliferation. Thus, the reduced expression of survivin, c-Myc and D-series cyclins by TQ is associated with the induction of apoptosis by this compound. Cyclins form active complexes with cyclindependent kinases, which are inhibited by cell cycle inhibitory proteins p27 and p21 (47). The elevated expression of p27 and $\mathrm{p} 21$ by TQ suggests that the compound can block cell cycle progression. In fact, TQ has been shown to induce G1 phase cell cycle arrest through the induction of p53 and p21 in HCT116 cells (10).

The STAT3 activation mechanism involves the phosphorylation of its tyrosine-705 and serine-727 residues followed by the formation of STAT3 dimer, which translocates to the nucleus and interacts with the promoter region of target genes (24). Although we have found that TQ can inhibit STAT3 tyrosine phosphorylation, the possible inhibitory effect of TQ on STAT3 serine phosphorylation merits further investigation. Several upstream kinases including EGFR tyrosine kinase (22), JAK2 (19) and Src (20) have been reported to activate STAT3. In the present study, we reported the novel finding that TQ treatment attenuated the constitutive phosphorylation of EGFR at tyrosine-1173 residue, JAK2 and Src kinases in HCT116 cells. We confirmed the roles of these kinases in STAT3 activation in HCT116 cells. Treatment of cells with specific pharmacological inhibitors of JAK2 and Src diminished the phosphorylation of EGFR and STAT3, while the blockade of EGFR by gefitinib inhibited STAT3 phosphorylation at tyrosine-705 residue without affecting that of JAK2 and Src. These findings suggest that JAK 2 and Src regulate STAT3 activation via phosphorylation of EGFR in HCT116 cells.

Our findings are in accordance with a previous study by Nourazarian et al (48), who demonstrated that combined EGFR and c-Src antisense deoxyoligonucleotides inhibited the proliferation of human colon cancer (HT29) cells. It has been reported that the blockade of Src kinase by PP2 attenuates EGFR phosphorylation at both tyrosine-845 and tyrosine-1173 residues in cervical cancer cells. According to this study, the phosphorylation of EGFR at tyrosine-1173, but not tyrosine-845, is involved in the proliferation of cervical cancer cells (27). The distinct roles of different EGFR tyrosine phosphorylation in colon cancer cell proliferation have not yet been clarified and the effect of TQ on EGFR phosphorylation at other tyrosine residues warrants further examination. The activation of Src also phosphorylates STAT3 at tyrosine-705 residue and contributes to colon cancer cell proliferation (20). Besides Src, the upstream kinase JAK2 has been shown to transactivate EGFR as the treatment of cells with JAK2 inhibitor AG490 attenuated EGFR phosphorylation in human epidermoid carcinoma cells (26). In agreement with this study, we found that JAK2 inhibition by AG490 diminished EGFR phosphorylation in HCT116 cells and TQ attenuated the activation of both JAK2 and EGFR. Since combined inhibition of EGFR and STAT3 have been shown to be more effective in suppressing growth of various cancer cells $(26,49)$, the ability 
of TQ to inhibit both EGFR and STAT3, hence, appears as a mechanistic basis of its antitumor effects in colon cancer cells.

\section{Acknowledgements}

The present study was funded by the Settlement Research Grant of Keimyung University in 2011 allocated to Kyung-Soo Chun.

\section{References}

1. Jemal A, Center MM, DeSantis C and Ward EM: Global patterns of cancer incidence and mortality rates and trends. Cancer Epidemiol Biomarkers Prev 19: 1893-1907, 2010.

2. Jemal A, Siegel R, Xu J and Ward E: Cancer statistics, 2010. CA Cancer J Clin 60: 277-300, 2010.

3. Merika E, Saif MW, Katz A, Syrigos K and Morse M: Review. Colon cancer vaccines: an update. In Vivo 24: 607-628, 2010.

4. Chung MY, Lim TG and Lee KW: Molecular mechanisms of chemopreventive phytochemicals against gastroenterological cancer development. World J Gastroenterol 19: 984-993, 2013.

5. Banerjee S, Padhye S, Azmi A, et al: Review on molecular and therapeutic potential of thymoquinone in cancer. Nutr Cancer 62 : 938-946, 2010.

6. Kundu J, Kim DH, Kundu JK and Chun KS: Thymoquinone induces heme oxygenase-1 expression in $\mathrm{HaCaT}$ cells via Nrf2/ ARE activation: Akt and AMPK $\alpha$ as upstream targets. Food Chem Toxicol 65: 18-26, 2014.

7. Kundu JK, Liu L, Shin JW and Surh YJ: Thymoquinone inhibits phorbol ester-induced activation of NF- $\mathrm{KB}$ and expression of COX-2, and induces expression of cytoprotective enzymes in mouse skin in vivo. Biochem Biophys Res Commun 438: 721-727, 2013.

8. Lei X, Lv X, Liu M, et al: Thymoquinone inhibits growth and augments 5-fluorouracil-induced apoptosis in gastric cancer cells both in vitro and in vivo. Biochem Biophys Res Commun 417: 864-868, 2012.

9. Yi T, Cho SG, Yi Z, et al: Thymoquinone inhibits tumor angiogenesis and tumor growth through suppressing AKT and extracellular signal-regulated kinase signaling pathways. Mol Cancer Ther 7: 1789-1796, 2008.

10. Gali-Muhtasib H, Diab-Assaf M, Boltze C, et al: Thymoquinone extracted from black seed triggers apoptotic cell death in human colorectal cancer cells via a p53-dependent mechanism. Int J Oncol 25: 857-866, 2004

11. Gali-Muhtasib H, Ocker M, Kuester D, et al: Thymoquinone reduces mouse colon tumor cell invasion and inhibits tumor growth in murine colon cancer models. J Cell Mol Med 12: 330-342, 2008

12. Jafri SH, Glass J, Shi R, Zhang S, Prince M and KleinerHancock $\mathrm{H}$ : Thymoquinone and cisplatin as a therapeutic combination in lung cancer: In vitro and in vivo. J Exp Clin Cancer Res 29: 87, 2010.

13. Kaseb AO, Chinnakannu K, Chen D, et al: Androgen receptor and E2F-1 targeted thymoquinone therapy for hormone-refractory prostate cancer. Cancer Res 67: 7782-7788, 2007.

14. Jrah-Harzallah H, Ben-Hadj-Khalifa S, Almawi WY, Maaloul A, Houas Z and Mahjoub T: Effect of thymoquinone on 1,2-dimethylhydrazine-induced oxidative stress during initiation and promotion of colon carcinogenesis. Eur J Cancer 49: 1127-1135, 2013.

15. Hanahan D and Weinberg RA: Hallmarks of cancer: the next generation. Cell 144: 646-674, 2011.

16. Burz C, Berindan-Neagoe I, Balacescu O and Irimie A: Apoptosis in cancer: key molecular signaling pathways and therapy targets. Acta Oncol 48: 811-821, 2009.

17. Martin SJ and Green DR: Protease activation during apoptosis: death by a thousand cuts? Cell 82: 349-352, 1995.

18. Ihle JN: The Stat family in cytokine signaling. Curr Opin Cell Biol 13: 211-217, 2001.

19. Slattery ML, Lundgreen A, Kadlubar SA, Bondurant KL and Wolff RK: JAK/STAT/SOCS-signaling pathway and colon and rectal cancer. Mol Carcinog 52: 155-166, 2013.

20. Laird AD, Li G, Moss KG, et al: Src family kinase activity is required for signal tranducer and activator of transcription 3 and focal adhesion kinase phosphorylation and vascular endothelial growth factor signaling in vivo and for anchorage-dependent and -independent growth of human tumor cells. Mol Cancer Ther 2: 461-469, 2003
21. Berclaz G, Altermatt HJ, Rohrbach V, Siragusa A, Dreher E and Smith PD: EGFR dependent expression of STAT3 (but not STAT1) in breast cancer. Int J Oncol 19: 1155-1160, 2001.

22. Chan KS, Carbajal S, Kiguchi K, Clifford J, Sano S and DiGiovanni J: Epidermal growth factor receptor-mediated activation of Stat 3 during multistage skin carcinogenesis. Cancer Res 64: 2382-2389, 2004.

23. Aggarwal BB, Kunnumakkara AB, Harikumar KB, et al: Signal transducer and activator of transcription-3, inflammation, and cancer: how intimate is the relationship? Ann NY Acad Sci 1171: 59-76, 2009.

24. Johnston PA and Grandis JR: STAT3 signaling: anticancer strategies and challenges. Mol Interv 11: 18-26, 2011.

25. Masuda M, Suzui M, Yasumatu R, et al: Constitutive activation of signal transducers and activators of transcription 3 correlates with cyclin D1 overexpression and may provide a novel prognostic marker in head and neck squamous cell carcinoma. Cancer Res 62: 3351-3355, 2002.

26. Dowlati A, Nethery D and Kern JA: Combined inhibition of epidermal growth factor receptor and JAK/STAT pathways results in greater growth inhibition in vitro than single agent therapy. Mol Cancer Ther 3: 459-463, 2004.

27. Kong L, Deng Z, Shen H and Zhang Y: Src family kinase inhibitor PP2 efficiently inhibits cervical cancer cell proliferation through down-regulating phospho-Src-Y416 and phospho-EGFR-Y1173. Mol Cell Biochem 348: 11-19, 2011.

28. Kopetz S: Targeting SRC and epidermal growth factor receptor in colorectal cancer: rationale and progress into the clinic. Gastrointest Cancer Res 1: S37-S41, 2007.

29. Mann JR, Backlund MG and DuBois RN: Mechanisms of disease: Inflammatory mediators and cancer prevention. Nat Clin Pract Oncol 2: 202-210, 2005.

30. Woo CC, Kumar AP, Sethi G and Tan KH: Thymoquinone: potential cure for inflammatory disorders and cancer. Biochem Pharmacol 83: 443-451, 2012.

31. El-Najjar N, Chatila M, Moukadem H, et al: Reactive oxygen species mediate thymoquinone-induced apoptosis and activate ERK and JNK signaling. Apoptosis 15: 183-195, 2010.

32. Gali-Muhtasib H, Kuester D, Mawrin C, et al: Thymoquinone triggers inactivation of the stress response pathway sensor CHEK1 and contributes to apoptosis in colorectal cancer cells. Cancer Res 68: 5609-5618, 2008.

33. Koka PS, Mondal D, Schultz M, Abdel-Mageed AB and Agrawal KC: Studies on molecular mechanisms of growth inhibitory effects of thymoquinone against prostate cancer cells: role of reactive oxygen species. Exp Biol Med 235: 751-760, 2010.

34. Hussain AR, Uddin S, Ahmed M, Al-Dayel F, Bavi PP and Al-Kuraya KS: Phosphorylated I $\kappa \mathrm{B} \alpha$ predicts poor prognosis in activated B-cell lymphoma and its inhibition with thymoquinone induces apoptosis via ROS release. PLoS One 8: e60540, 2013.

35. Valassiadou KE, Stefanaki K, Tzardi M, et al: Immunohistochemical expression of p53, bcl-2, mdm2 and waf1/p21 proteins in colorectal adenocarcinomas. Anticancer Res 17: 2571-2576, 1997.

36. Bonnotte B, Favre N, Moutet M, et al: Bcl-2-mediated inhibition of apoptosis prevents immunogenicity and restores tumorigenicity of spontaneously regressive tumors. J Immunol 161: 1433-1438, 1998.

37. Dobi E, Monnien F, Kim S, et al: Impact of STAT3 phosphorylation on the clinical effectiveness of anti-EGFR-based therapy in patients with metastatic colorectal cancer. Clin Colorectal Cancer 12: 28-36, 2013.

38. Corvinus FM, Orth C, Moriggl R, et al: Persistent STAT3 activation in colon cancer is associated with enhanced cell proliferation and tumor growth. Neoplasia 7: 545-555, 2005.

39. Lin Q, Lai R, Chirieac LR, et al: Constitutive activation of JAK3/ STAT3 in colon carcinoma tumors and cell lines: inhibition of JAK3/STAT3 signaling induces apoptosis and cell cycle arrest of colon carcinoma cells. Am J Pathol 167: 969-980, 2005.

40. Badr G, Mohany M and Abu-Tarboush F: Thymoquinone decreases F-actin polymerization and the proliferation of human multiple myeloma cells by suppressing STAT3 phosphorylation and Bcl2/Bcl-XL expression. Lipids Health Dis 10: 236-243, 2011.

41. Li F, Rajendran P and Sethi G: Thymoquinone inhibits proliferation, induces apoptosis and chemosensitizes human multiple myeloma cells through suppression of signal transducer and activator of transcription 3 activation pathway. $\mathrm{Br} \mathrm{J}$ Pharmacol 161: 541-554, 2010 
42. Zhang X, Liu P, Zhang B, Mao H, Shen L and Ma Y: Inhibitory effects of STAT3 decoy oligodeoxynucleotides on human epithelial ovarian cancer cell growth in vivo. Int J Mol Med 32: 623-628, 2013.

43. Kanda N, Seno H, Konda Y, et al: STAT3 is constitutively activated and supports cell survival in association with survivin expression in gastric cancer cells. Oncogene 23: 4921-4929, 2004.

44. Asanuma K, Tsuji N, Endoh T, Yagihashi A and Watanabe N: Survivin enhances Fas ligand expression via up-regulation of specificity protein 1-mediated gene transcription in colon cancer cells. J Immunol 172: 3922-3929, 2004.

45. Lin L, Liu A, Peng Z, et al: STAT3 is necessary for proliferation and survival in colon cancer-initiating cells. Cancer Res 71: 7226-7237, 2011.

46. Forgue-Lafitte ME, Coudray AM, Breant B and Mester J: Proliferation of the human colon carcinoma cell line HT29: autocrine growth and deregulated expression of the c-myc oncogene. Cancer Res 49: 6566-6571, 1989.
47. Abukhdeir AM and Park BH: P21 and p27: roles in carcinogenesis and drug resistance. Expert Rev Mol Med 10: e19, 2008.

48. Nourazarian AR, Najar AG, Farajnia S, Khosroushahi AY, Pashaei-Asl R and Omidi Y: Combined EGFR and c-Src antisense oligodeoxynucleotides encapsulated with PAMAM Denderimers inhibit HT-29 colon cancer cell proliferation. Asian Pac J Cancer Prev 13: 4751-4756, 2012.

49. Boehm AL, Sen M, Seethala R, et al: Combined targeting of epidermal growth factor receptor, signal transducer and activator of transcription-3, and $\mathrm{Bcl}-\mathrm{X}(\mathrm{L})$ enhances antitumor effects in squamous cell carcinoma of the head and neck. Mol Pharmacol 73: 1632-1642, 2008. 